\title{
Analysis of subcellular metabolite levels of potato tubers (Solanum tuberosum) displaying alterations in cellular or extracellular sucrose metabolism
}

\author{
Eva M. Farre · Alisdair R. Fernie • \\ Lothar Willmitzer
}

Received: 9 October 2007/ Accepted: 1 February 2008/Published online: 27 February 2008

(C) Springer Science+Business Media, LLC 2008

\begin{abstract}
The expression of a heterologous invertase in potato tubers (Solanum tuberosum) in either the cytosol or apoplast leads to a decrease in total sucrose content and to an increase in glucose. Depending on the targeting of the enzyme different changes in phenotype and metabolism of the tubers occur: the cytosolic invertase expressing tubers show an increase in the glycolytic flux, accumulation of amino acids and organic acids, and the appearance of novel disaccharides; however, these changes are not observed when the enzyme is expressed in the apoplast [Roessner et al. (2001). Plant Cell, 13, 11-29]. The analysis of these lines raised several questions concerning the regulation of compartmentation of metabolites in potato tubers. In the current study we addressed these questions by performing comparative subcellular metabolite profiling. We demonstrate that: (i) hexoses accumulate in the vacuole independently of their site of production, but that the cytosolic invertase expression led to a strong increase in the cytosolic glucose concentration and decrease in cytosolic sucrose, whereas these effects were more moderate in the apoplastic expressors; (ii) three out of four of the novel compounds found in the cytosolic overexpressors
\end{abstract}

Electronic supplementary material The online version of this article (doi:10.1007/s11306-008-0107-5) contains supplementary material, which is available to authorized users.

E. M. Farre $(\bowtie) \cdot$ A. R. Fernie $~$ L. Willmitzer

Max-Planck-Institut für Molekulare Pflanzenphysiologie,

Am Mühlenberg 1, 14476 Potsdam-Golm, Germany

e-mail: efarre@ucsd.edu

Present Address:

E. M. Farre

Department of Developmental and Cell Biology,

University of California, San Diego, 9500 Gilman Drive,

La Jolla, CA 92093, USA accumulate in the same compartment; (iii) despite changes in absolute cellular content the subcellular distribution of amino acids was invariant in the invertase overexpressing tubers. These results are discussed in the context of current models of the compartmentation of primary metabolism in heterotrophic plant tissues.

Keywords Compartmentation ·

Plant heterotrophic carbon metabolism .

Maltose biosynthesis - Metabolite profiling
Abbreviations
Glc-6-P glucose-6-phosphate
GABA $\quad \gamma$-aminobutyric acid
Fru-6-P fructose-6-phosphate
3-P-glycerate 3-phosphoglycerate

\section{Introduction}

In potato, a switch occurs in the sucrolytic pathway as elongating stolons begin to form tubers. Sucrose synthase activity markedly increases during tuberization whereas acid invertase levels decrease (Fernie and Willmitzer 2001). To study the role of the mechanism of sucrose breakdown in starch accumulation in potato tubers, transgenic approaches involving the ectopic expression of a yeast invertase under the control of the tuber-specific patatin promoter were adopted (Sonnewald et al. 1997). The heterologous invertase was targeted to either the cytosol or the apoplast. The fact that sucrose levels were strongly reduced in tubers expressing either of these constructs suggests that sucrose is available to the apoplast even at later stages of tuber development. Both cytosolic and 
apoplastic invertase expressors also showed large increases in glucose levels but surprisingly a reduced starch content. Strikingly, however, differences in the localization of the enzyme had profound effects on both the phenotype and on biochemical characteristics of the tubers other than those described above. The apoplastic expression of invertase (U-IN1 lines) led to larger tubers, but a reduction in the numbers of tubers per plant. In contrast, the cytosolic invertase lines (U-IN2) developed a higher number of small tubers (Sonnewald et al. 1997). Further analysis of these lines showed that U-IN2 had strong increases in the content of hexose phosphates and in the rates of glycolysis and respiration (Trethewey et al. 1998; Hajirezaei et al. 2000; Bologa et al. 2003). Application of GC-MS metabolite profiling in the U-IN2 lines has revealed that these tubers exhibited elevated contents of organic and amino acids. Surprisingly, with the exception of a moderate increase in hexose phosphates, none of these changes were observed in the apoplastic invertase-expressing lines (Hajirezaei et al. 2000; Roessner et al. 2001). A further characteristic of the cytosolic expressing tubers is the increased levels of disaccharides such as maltose, isomaltose and trehalose, metabolites that were not detectable by GC-MS analysis of the wild type lines. However, only maltose, the most abundant of these compounds, is detectable in apoplastic invertase tubers (Roessner et al. 2001).

In recent years focus on this much-studied plant enzyme has been largely dedicated to cloning the corresponding genes from an ever expanding list of species, identifying proteins that influence invertase activity by studying protein structure-function relationships, and in linking invertase activity to physiological function (for a review see Lytovchenko et al. 2007). However, relatively little study has been concentrated on improving our understanding of the compartmentation of sugar metabolism. Previous studies of invertase expressing tubers have, however, raised many questions on the importance and role of compartmentation on tuber metabolism. The first concerns the subcellular localization of sugars in the tubers. Since both lines display a decrease in sucrose and an increase in glucose of similar magnitude, differences in their subcellular levels have been hypothesized to explain the biochemical differences between these lines (Sonnewald et al. 1997; Fernie et al. 2000, Hajirezaei et al. 2000). It has been previously postulated that compartmentation of metabolites may mediate this effect either by simple metabolic effects or by carbon signalling mechanisms (Fernie et al. 2001). However, there is little experimental evidence to support the latter postulate. Second, as mentioned above, one striking difference between apoplastic and cytosolic invertase expressing tubers is the appearance of several novel disaccharides such as maltose, isomaltose and trehalose, metabolites that were not detectable by GC-MS analysis of the wild type lines (Roessner et al. 2001). These compounds were previously postulated to result from an enhanced rate of breakdown of tuber starch or to be diagnostic of an activation of signalling processes (Roessner et al. 2001). Despite the recent identification of a plastidial maltose transporter and subsequent independent support for the importance of this protein in leaf starch degradation (Chia et al. 2004), its role in heterotrophic tissue is currently unknown. However, recent stable isotope labelling studies suggest that little of the maltose in the cytosolic invertase expressing lines is likely to be derived via the process of starch degradation (Roessner-Tunali et al. 2004). In recent years an important role for maltose in conferring cold tolerance has emerged (Kaplan and Guy 2005), hence a more detailed understanding of its biosynthesis and degradative pathways could additionally be of high importance from an applied perspective. For this reason we intended to evaluate the subcellular metabolite profiles in order to gain information on the distribution and potential metabolism of these compounds using a nonaqueous fractionation technique coupled to gas-chromatography mass-spectrometry (Farre et al. 2001; Fernie et al. 2004). The data obtained from this study provide a relatively comprehensive picture of the compartmentation of sugar metabolism within the tuber and provide several important further insights into the role of metabolic compartmentation in metabolic regulation.

\section{Methods}

\subsection{Plant material and growth conditions}

Solanum tuberosum L. cv. Desiree was supplied by Saatzucht Lange AG (Bad Schwartau, Germany). The line U-IN2-30 expresses a yeast invertase under the control of the patatin-B33 promoter (Sonnewald et al. 1997). The line U-IN1-33 expresses a yeast invertase, also under the control of the patatin-B33 promoter, with an untranslated leader sequence that allows apoplastic targeting (Sonnewald et al. 1997). Plants were grown from stem cuttings. The plants used for biochemical analysis were raised in the greenhouse in 21 pots under a $16 \mathrm{~h}$ light, $8 \mathrm{~h}$ dark regime at $22^{\circ} \mathrm{C}$ with supplementary light to ensure a minimum of $250 \mu \mathrm{mol}$ photons $\mathrm{m}^{-2} \mathrm{~s}^{-1}$. For sampling of tuber material, a cylinder (12 $\mathrm{mm}$ diameter) was cut perpendicular to the stolon-apex axis in the middle of the tuber (Merlo et al. 1993). For biochemical analysis tuber slices $1 \mathrm{~mm}$ thick were cut from the cylinder and immediately frozen in liquid nitrogen and stored at $-80^{\circ} \mathrm{C}$ until use. 


\subsection{Chemicals}

The biochemical enzymes were purchased from Roche (Mannheim, Germany). All chemicals were obtained from Roche (Mannheim, Germany), Sigma (München, Germany), or Merck (Darmstadt, Germany).

\subsection{Non-aqueous fractionation of tuber tissue}

Tuber tissue was prepared and fractionated using a nonaqueous fractionation method as described in Farré et al. (2001). Previously described potato plants displaying tuber specific expression of a yeast-derived invertase either targeted to the cell wall (U-IN1; hereafter called apoplast invertase overexpressors) or expressed in the absence of a targeting sequence (U-IN2 hereafter called cytosolic invertase overexpressors) were grown alongside wild type controls. Given the labor intensity of the work a single welldescribed representative line per genotype was grown in each case (U-IN1-33 and U-IN2-30; Sonnewald et al. 1997; Trethewey et al. 1998; Roessner et al. 2001; UrbanczykWochniak et al. 2003; Roessner-Tunali et al. 2004). As a preliminary experiment we confirmed that the lines retained similar invertase activities to those already published (data not shown). Having confirmed this fact, we next extracted samples from 10-week-old greenhouse grown potato tubers of wild type and invertase expressing lines and subjected these to a non-aqueous fractionation method that had been previously optimised for potato tubers (Farre et al. 2001). The mean percentage distributions of pyrophosphatase and ADP-glucose pyrophosphorylase activities represent the plastidial marker, those of UDP-glucose pyrophosphorylase and pyrophoshate-dependent phosphofructokinase the cytosolic marker and $\alpha$-mannosidase the vacuolar marker. Since mitochondrial markers co-fractionate with the cytosolic markers, it is not possible to resolve these two compartments using this method. Data for the wild type represents the average of 5 independent fractionations. Two of these fractionations were performed using tubers from the same plant, and each of the other 3 used tubers from a different plant (i.e. 4 different plants were used). In the case of the U-IN1-33 samples, data represents the average of 4 independent fractionations. Two of these fractionations were performed using tubers from the same plant, and each of the other two used tubers from a different plant. In the case of the U-IN2-30 samples, data represents the average of 4 independent fractionations; each of them performed using tubers from a different plant. Although all tuber samples were collected from 10-week-old plants, tuber size differed depending on the localization of the overexpressed invertase, as previously described, with tubers from the line U-IN2-30 being significantly smaller than wild type or U-IN1-33 tubers (Sonnewald et al. 1997).

\subsection{Determination of enzyme activities}

Extraction of enzyme activities was performed exactly as described in Farré et al. (2001). Invertase activities were determined as described by Hajirezaei et al. (2000).

\subsection{Determination of metabolic intermediates}

Metabolite levels in the fractionated material were analysed by gas-chromatography mass-spectrometry (GC-MS) in methanol extracts as described by Roessner et al. (2001). This methodology allows the detection of selected free amino acids, organic acids, sugars, sugar phosphates, and sugar alcohols. We focussed here only on the 50 metabolites that we could unambiguously identify. Limits of detection correspond to nanomolar levels per gram fresh weight depending on the metabolite in question. The technique additionally affords a wide dynamic range approaching four orders of magnitude for some metabolites. (Kopka 2006).

\subsection{Data analysis}

A three-compartment calculation program (Bestfit) that has been described in detail by Riens et al. (1991), was used to evaluate subcellular metabolite distributions. Absolute concentrations were calculated using the metabolite total tissue content, the metabolite relative distribution and the estimations of subcellular volumes from Farré et al. (2001): cytosol + mitochondria, $0.11 \mathrm{ml} \mathrm{g}$ fresh weight ${ }^{-1}$; plastid $0.13 \mathrm{ml} \mathrm{g}$ fresh weight ${ }^{-1}$ and vacuole $0.58 \mathrm{ml} \mathrm{g}$ fresh weight ${ }^{-1}$.

\section{Results}

\subsection{Variability of the non-aqueous fractionation procedure}

The non-aqueous fractionation technique relies on the cofractionation of metabolites with compartment specific enzymatic activities, and on the subsequent deconvolution of the subcellular distribution based on correlation analysis. The relatively low resolution of this technique is mainly due to the large particle size of the homogenized tissue to be fractionated, which leads to compartments co-fractionating with each other. This is more critical for small compartments such as the cytosol than for larger ones such as the vacuole, since there will be particles that will contain exclusively vacuolar material. Due to this fact the deconvolution analysis plays a key role in this technique. Due to the variability of the procedure we only analysed 50 metabolites that we could unambiguously identify in all fractions. In order to estimate the variability of this fractionation procedure we 


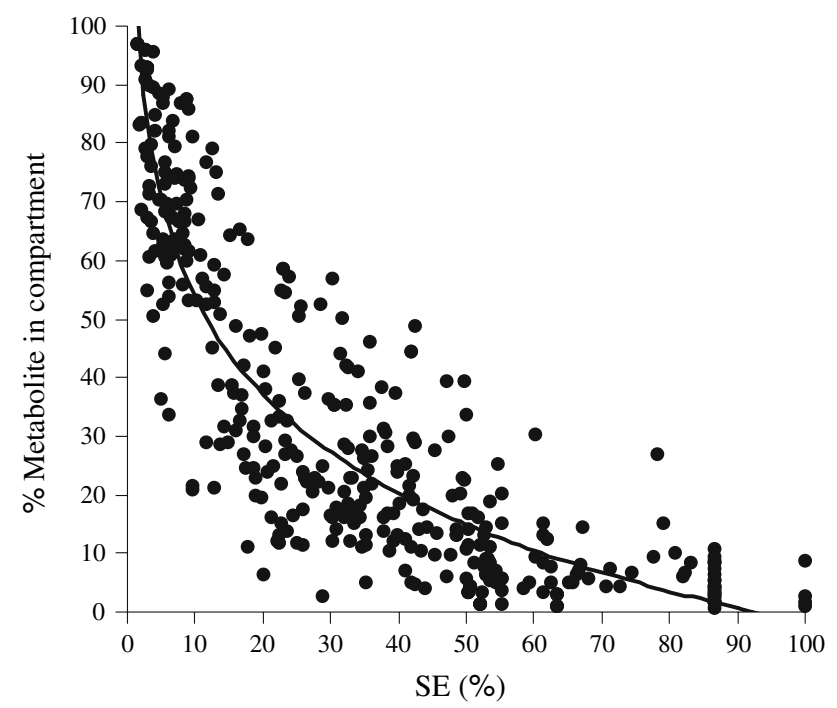

Fig. 1 Variability of metabolite subcellular distributions. The mean relative distributions (in percent) from all measurements shown in Supplementary Tables 1 are 2 are plotted against their standard error (SE) in percent

plotted the relative metabolite content in each compartment against its standard error. Figure 1 shows that the variability in the determination of a particular subcellular localization increases with decreasing metabolite content in that particular compartment. The average standard error is $34 \%$, which increases rapidly with relative contents smaller than $30 \%$.

\subsection{Subcellular distribution and concentrations of sugar and sugar alcohols in cytosolic and apoplastic invertase expressors}

The relative subcellular distribution and absolute concentration for each metabolite were calculated as described in detail in Farré et al. (2001) and in Methods Sect. 2.6. Perhaps unsurprisingly, the distribution of glucose and sucrose were markedly altered in the invertase expressing lines (Fig. 2). Cytosolic invertase overexpressing tubers had their glucose content equally distributed between the vacuole and the cytosol (Fig. 2a). By contrast, in the lines in which invertase expression was targeted to the apoplast, the subcellular distribution of glucose mirrored that observed in the wild type (Fig. 1a; Table 1, Supplementary Table 1). Thus cytosolic expression of invertase results in a distinctive subcellular distribution, and concentration, of sugars than apoplastic expression. Dramatic changes were also observed in the subcellular distribution of sucrose. Whereas in wild type tubers sucrose is present predominantly in the vacuole, in the cytosolic invertase-expressing tubers sucrose was nearly equally distributed among the amyloplast, cytosol, and vacuole (Fig. 2b). This trend became even more extreme in apoplastic invertase expressors, where the relative levels of sucrose present in the vacuole were even smaller, leading to the preferential localization of sucrose in the cytosol (Fig. 2b). With respect to fructose, the relative subcellular distribution was unaltered irrespective of genotype with more than $70 \%$ of fructose associated with the vacuole in both invertase expressing and wild type tubers (Supplementary Table 1). Thus cytosolic expression of invertase results in a distinctive subcellular distribution, and concentration, of sugars than apoplastic expression.

The changes in distribution of sucrose, glucose and fructose are reflected in changes in the estimated subcellular concentrations of both lines (Table 1). In wild type tubers, fructose concentration is higher in the vacuole than in the cytosol. Although the overall fructose concentration was elevated in the apoplastic invertase overexpressor about 80fold, this relationship was maintained. In contrast, in the cytosolic invertase overexpressor line vacuolar and cytosolic fructose concentrations were similar. Glucose concentration was 1.5-2-fold higher in the cytosol when compared to the vacuole in both wild type and apoplastic invertase overexpressors, but more that 5-fold higher in the cytosolic invertase overexpressor tubers. Both U-IN2 and U-IN1 lines displayed a marked decrease in cytosolic and vacuolar sucrose concentrations. Interestingly in both cases the decrease was stronger in the vacuole than in the cytosol.

Line UIN2-30 has previously been documented to contain detectable levels of several metabolites that are below the level of detection in wild type tubers (Roessner et al. 2001). Further experimentation revealed that these probably result from side reactions due to increased cytosolic glucose concentrations since their levels are depleted on reduction of the glucose content following supertransformation of this line with a second transgene (UrbanczykWochniak et al. 2003; Farre et al. 2006). The trehalose and maltose, present in the cytosolic invertase-expressors were localized in the cytosol, whereas isomaltose was preferentially localized in the vacuole and maltitol seemed to be more or less evenly distributed throughout the cell (Fig. 2c).

Despite the broad changes reported above for the distributions and concentrations of sugars, few differences were observed in the subcellular concentrations of sugarderivatives. These were mainly confined to increases in the cytosolic concentration of Glc-6-P and Fru-6-P, 3-P-glycerate and in the overall mannitol concentration in line UIN2-30. Interestingly, there was a significant change in the subcellular distribution of inositol in the cytosolic invertase overexpressor but not in the apoplastic overexpressor. Inositol has previously been reported to be largely associated with the vacuole in wild type tubers (Farre et al. 2001). However, inositol was found to be mainly associated with the cytosol in line U-IN2 (Fig. 2d). 
Fig. 2 Subcellular metabolite distribution in wild type and transgenic tubers. The tissue was fractionated using a nonaqueous procedure. Metabolites in each fraction were measured in methanol extracts using GC-MS. The subcellular distributions were calculated by comparing the metabolite and marker enzyme distributions using a three-compartment calculation program. Results represent the means $\pm \mathrm{SE}$ of measurements of four different fractionations with different tuber samples from U-IN2-30 and U-IN1-33 and the results of five different fractionations with different tuber samples from the wild type
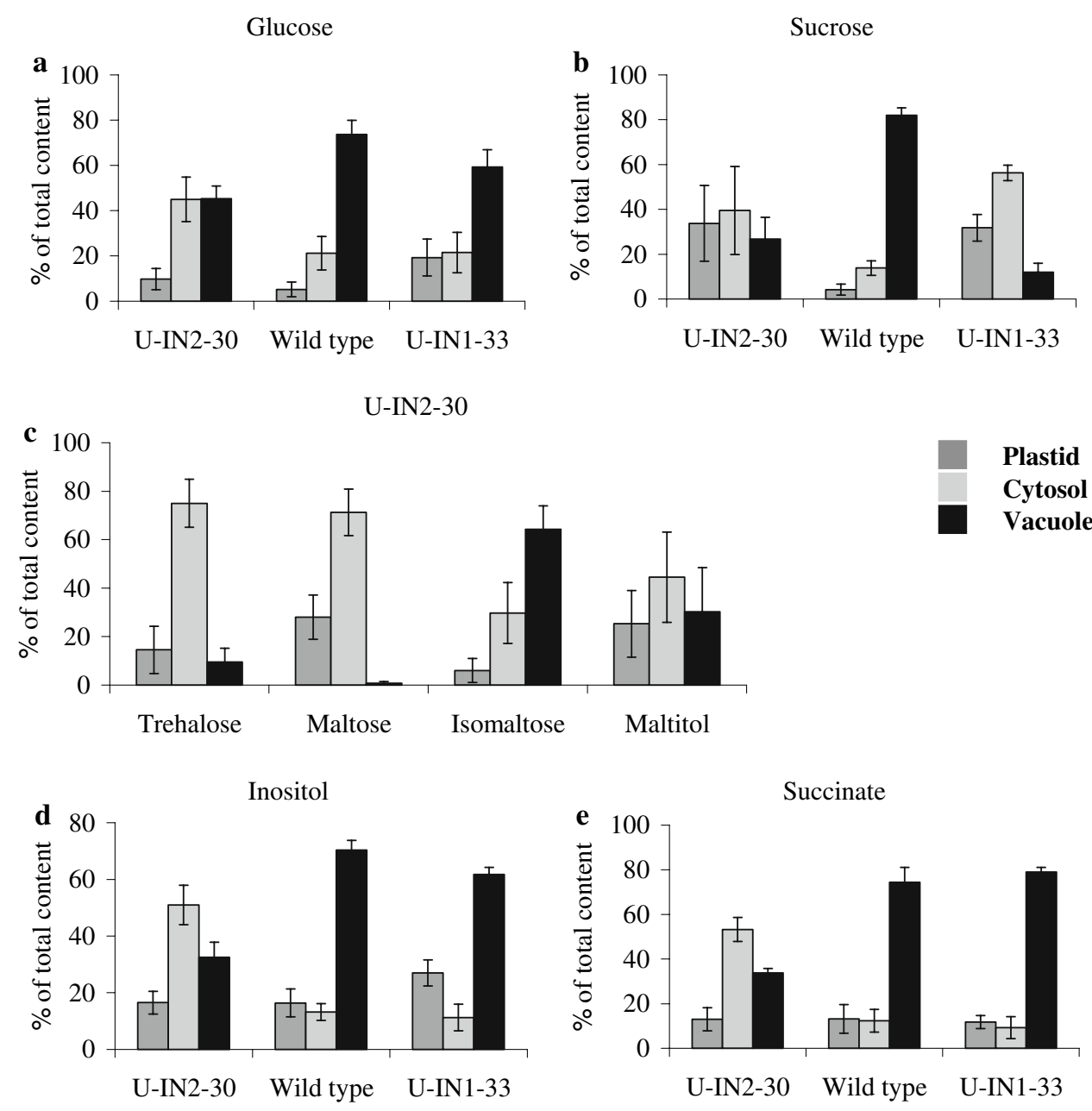

\subsection{Subcellular distribution and concentrations} of amino acids and organic acids in cytosolic and apoplastic invertase expressors

Previous studies on wild type tubers have indicated that the highest distribution of organic acids and amino acids is in the vacuole (Farre et al. 2001). However, the large volume of the vacuole serves to dilute their overall concentration. Thus, on a millimolar basis, the concentration of organic and amino acids is actually higher in the cytosol. This was confirmed in our study on the invertase expressors (Tables 1, 2, Supplementary Tables 1 and 2). The majority of organic acids in all three lines accumulate preferentially in the vacuole. Exceptions include ascorbate, which is preferentially localized within the amyloplast and $\alpha$-ketoglutarate which is predominantly localised in the cytosolic/ mitochondrial compartment. Changes in the subcellular distribution of fumarate and succinate in the cytosolic invertase overexpressors were the only significant changes among organic acids in the transgenic tubers. In both cases the cytosolic content was increased and their vacuolar content reduced (Fig. 1e, Supplementary Table 2).
When the data was evaluated in terms of concentration the most dramatic change was again observed in cytosolic succinate, which increased ten-fold in line U-IN2-30 (Table 1). This change is a function both of an increase in the cellular content of succinate and a change in its distribution to favour compartmention within the cytosol (Fig. 1e). This line was also characterised by a reduction in vacuolar concentrations of isocitrate and increases in the cytosolic concentrations of fumarate and the cytosolic and vacuolar concentrations of malate. In contrast, line UIN133 displayed little changes in the levels of Krebs cycle intermediates with the exception of slightly reduced cytosolic concentrations of citrate and succinate.

Most amino acids displayed relatively similar patterns of distribution between the various genotypes. In both wild type and transgenic tubers, most amino acids were predominately localised to the vacuole (Supplementary Table 2). This contrasts with their predominantly cytosolic localization in leaves (Leidreiter et al. 1995; Benkeblia et al. 2007), which might be explained by the the storage function of vacuoles in heterotrophic tissues. In tubers the only exceptions are glutamate and aspartate that were also 


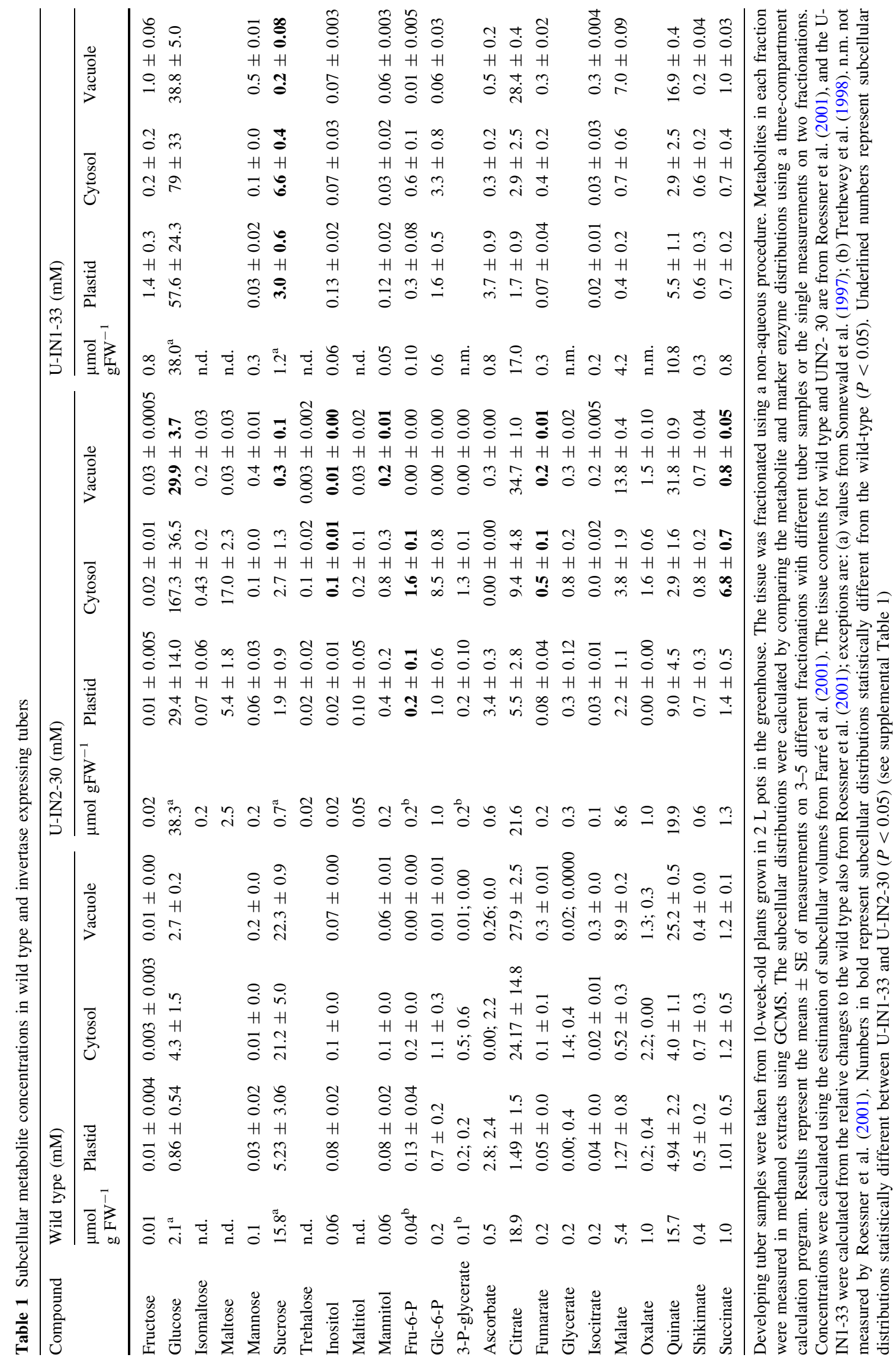




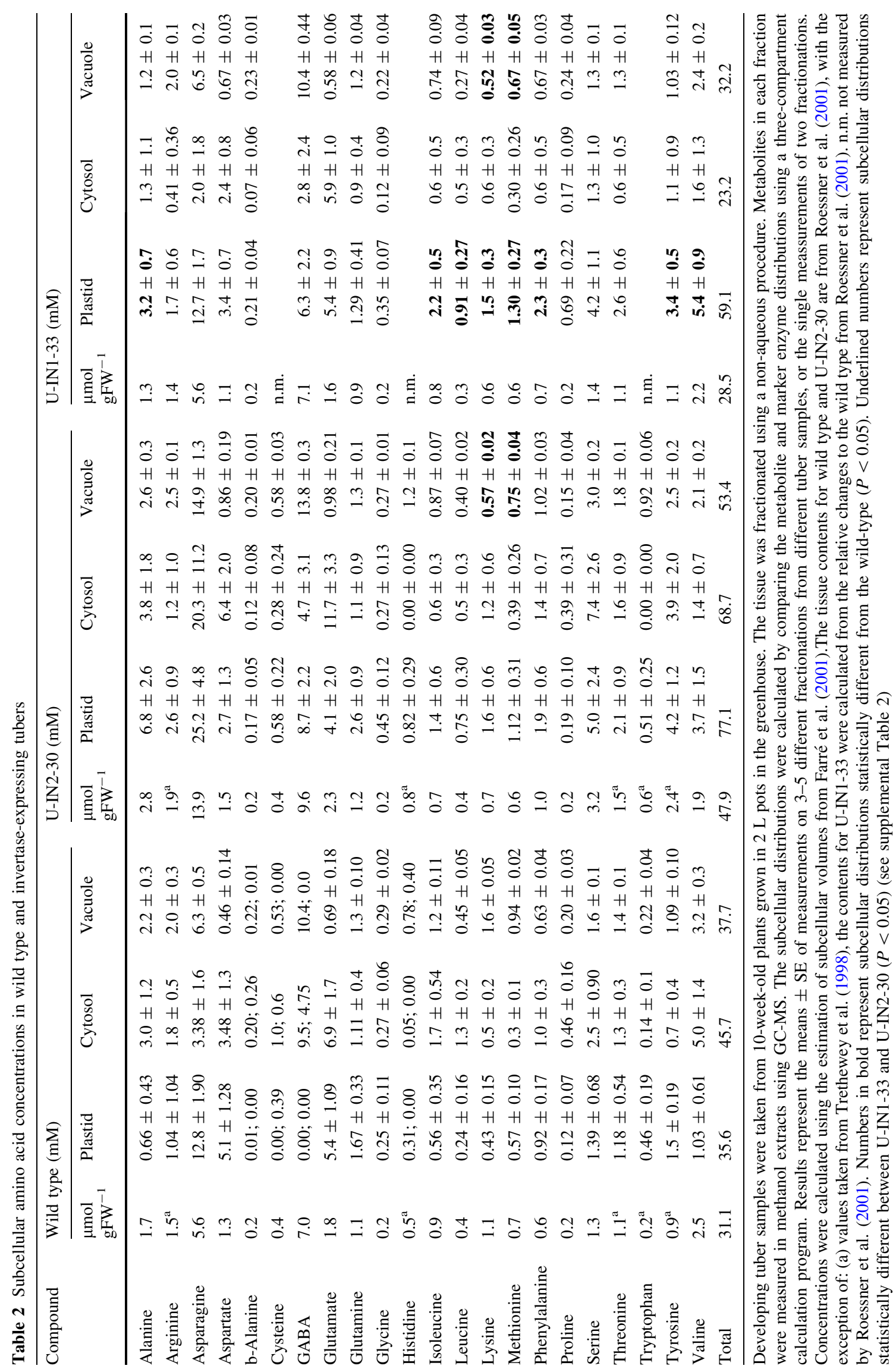


present at considerable amount in the amyloplast and cytosol (Supplemenatary Table 2). Whilst the general pattern of distribution is largely conserved across the genotypes about 11 amino acids display some modification of partitioning. On the one hand, in the cytosolic invertase overexpressor the vacuolar content of homoserine, lysine, methionine, 5-oxoproline was decreased, and the vacuolar content of tryptophane increased. On the other hand, the apoplastic invertase tubers displayed an increase in the plastidial content of alanine, isoleucine, leucine, lysine, methionine, phenylalanine, tryptophane, tyrosine and valine that correlated with a decrease in the vacuolar content of these amino acids.

The line U-IN2-30 but not the line U-IN1-33 displays an increase in total content of several major amino acids such as asparganie, GABA and glutamate (Roessner et al. 2001; Table 2). Since this increase did not correlate with subcelluar distribution changes, this overall increase led to higher concentrations in all compartments (Table 2, Supplementary Table 2). In contrast, the increase in overall tryptophane content was restricted to the vacuole due to the observed shift in subcellular distribution (Table 2). The apoplastic invertase overexpressors do not display major changes in total amino acid content (Roessener et al. 2001, Table 2). Therefore, the shifts in the subcellular distribution of several amino acids described previously led directly to changes in the subcellular concentrations of amino acids. In general there was an increase in the plastidial amino acid content in the U-IN1 tubers.

\section{Discussion}

An expanding research frontier in plant science is the enhancement of our understanding of the spatial resolution of biological processes. Here we adopted a non-aqueous fractionation method in order to enhance our understanding of the role of compartmentation of potato tuber sugar metabolism by analysing two thoroughly characterised transgenic lines exhibiting enhanced sucrose degradation in either the cytosol or apoplast. This method allows the subcellular analysis of large number of metabolites when combined with metabolite profiling techniques, enabling the identification of major shifts in subcellular distributions. The potato lines we investigate here, were initially created in a biotechnological attempt to increase tuber size and starch content, however, whilst the former was to some extent successful, the latter failed (Sonnewald et al. 1997). Comprehensive biochemical analysis of the lines revealed that the cytosolic lines were characterised by a massive increase in respiration and amino acid biosynthesis and a restriction of starch synthesis (Trethewey et al. 1998; Roessner et al. 2001; Roessner-Tunali et al. 2004). Further studies revealed that the stimulation in respiration was likely driven by the increased energy demands, made on the cell by the establishment of a massive sucrose futile cycle (Bologa et al. 2003), whilst the decrease in starch synthesis was at least in part due to a redox based inactivation of the reaction catalyzed by ADP-glucose phosphorylase (Tiessen et al. 2002). That said questions such as the availability of substrates to support biosynthesis and intracellular partitioning of metabolites in these lines were not previously addressed.

In this study we could show that there are indeed differences in both the relative distributions and subcellular concentrations of sucrose, glucose and fructose between cytosolic and apoplastic invertase-expressing tubers. In spite of similar increases in total glucose content, the cytosolic expressors have higher total levels of Glc-6-P than the apoplastic invertase expressors (Sonnewald et al. 1997; Hajirezaei et al. 2000; Roessner et al. 2000). However, there were no strong differences in the cytosolic glucose to Glc-6-P ratios between the two transgenic lines (20 and 24, for cytosolic and apoplastic expressors respectively). Thus, the availability of glucose in the cytosol appears to be the main reason for the smaller increase in hexose phosphates in U-IN1-33 in comparison to U-IN2-30. Compared to the wild type total fructose content increases only very little (up to 2-fold) in the cytosolic invertase expressors, but dramatically (up to 80fold) in the apoplastic invertase expressors (Sonnewald et al. 1997; Hajirezaei et al. 2000; Roessner et al. 2000). Both transgenic lines display strong decreases in the amount of total sucrose, mostly due to an up to 100 -fold reduction in the concentration of sucrose in the vacuole (Table 1). Cytosolic and apoplastic invertase expressing tubers significantly differ however, in their cytosolic sucrose concentration. In the cytosolic expressors sucrose is reduced to $20 \%$, in the apoplastic expressors it is reduced only to $50 \%$ of the concentration observed in wild type tubers. We were also able to reproduce our previous finding that a considerable amount of sucrose localized in the plastid (Table 1; Farré et al. 2001). However, whilst Gerrits et al. (2001) could show that the expression of an invertase in the plastids of potato tubers leads to a reduction in the total sucrose content, our results show that the expression of an invertase in either cytosol or apoplast did not lead to a reduction in plastidial sucrose concentration which is maintained at about $2 \mathrm{mM}$ (Table 1 ).

Analysing these data together allows us to propose the following model to explain the differences in sugar levels between the apoplastic and cytosolic invertase expressing tubers. In the cytosolic invertase expressing tubers almost all of the cytosolic sucrose is cleaved into glucose and fructose. Cytosolic fructose is immediately phosphorylated to Fru-6-P and consumed glycolytically. In contrast, most 
of the glucose remains unphosphorylated, since the hexokinase responsible for the phosphorylation of glucose is less efficient (Renz et al. 1993). Part of the resulting glucose pool is passively transported into the vacuole, since there is a clear concentration gradient from a high cytosolic to a low vacuolar concentration. In the apoplastic invertase expressing tubers, however, although hexoses are produced in the apoplast, glucose and fructose seem to accumulate in the vacuole (Table 1). It appears that any fructose present in the cytosol is very rapidly phosphorylated suggesting that fructose accumulating in the vacuole has been less available to the cytosolic fructokinase activity and thus, probably, has entered the vacuole via endocytosis (Etxeberria et al. 2005; Fernie et al. 2000). Such a connection would also explain the smaller increase in cytosolic glucose levels in the apoplastic invertase expressors as compared to the cytosolic expressors, in spite of the observation that in both transgenics the total tissue glucose rises to the same level. It would also explain the fact, that targeting of the yeast invertase to the potato tuber vacuole results in a very similar metabolic phenotype as to the apoplast (Junker et al. 2006). The direct measurements of subcellular metabolite concentrations presented here are thus consistent with earlier theories on sugar trafficking in potato tuber put forward independently by several groups (Etxeberria et al. 2005; Fernie et al. 2000).

Our studies confirmed earlier findings that levels of the disaccharides maltose, isomaltose and the sugar alcohol maltitol were elevated in the cytosolic invertase expressing lines (Roessner et al. 2001). Our non-aqueous fractionation approach allowed us to locate these metabolites in the cytosol alongside glucose, their potential biosynthetic precursor. That said recent studies on starch degradation and the pathway of cytosolic heteroglucan formation are in clear support of the importance of maltose export during starch degradation (Fettke et al. 2004, 2005; Weise et al. 2004), however, these studies are as yet largely restricted to photosynthesizing tissue. Some evidence, however, exists for starch turnover in the tuber (Sweetlove et al. 1996; Roessner-Tunali et al. 2004) and on the basis of ${ }^{13} \mathrm{C}$-glucose feeding studies we cannot exclude that this is the source of the maltose accumulation in line U-IN2-30 (Roessner-Tunali et al. 2004). Analysis of the subcellular distribution of the hexose phosphates in the cytosolic invertase expressors was also highly interesting since it suggests that the increased hexose phosphate production in the cytosol was not readily transported to the plastid. Previous non-aqueous fractionation studies (Farre et al. 2001, Tiessen et al. 2002), have suggested that the plastidial Glc-6-P transporter (Kammerer et al. 1998) was unlikely to limit starch synthesis in wild type lines. Our findings imply a greater role for this transporter in regulating starch synthesis than previously recognised.
With the exception of succinate, neither the cytosolic nor the apoplastic invertase expressor showed marked changes in the subcellular distribution of organic acids (Table 1). In cytosolic invertase-expressing tubers succinate was mainly associated with the cytosolic marker (Fig. 1e). In contrast, in the wild type and apoplastic invertase overexpressors, most succinate was localized in the vacuole, in agreement with our previous study (Farré et al. 2001). We still do not understand the reason for this observation. However, one plausible explanation might be an inhibition of succinate transport mechanisms, either out of the mitochondria or into the vacuole. It is, however, also conceivable that the strong increase in malate and the increase in the respiration rate of these tubers might influence succinate transport mechanisms.

Although only cytosolic invertases overexpressing tubers displayed an increase in total amino acid content $(48 \mu \mathrm{mol} g$ fresh weight ${ }^{-1}$ against $31 \mu \mathrm{mol} \mathrm{g}_{\text {fresh weight }}{ }^{-1}$ in wild type), both analysed transgenic lines exhibited an increase in cytosolic and plastidic amino acid concentration (Table 2). It thus seems that in potato tubers high rates of amino acid biosynthesis can be maintained in spite of high cytosolic and plastidic concentrations. In contrast, in leaves, both cytosolic and apoplastic expression led to decreased cytosolic and increased plastidial amino acid contents (Heineke et al. 1994).

In conclusion, the results presented here allowed the resolution of several important features of metabolic regulation in the potato tuber including the finding that the Gluc-6-P transporter appears to be limiting for starch synthesis under conditions of high substrate availability and the finding that the subcellular production site of glucose heavily influences the formation of tuber disaccharides. It seems likely, that in the next few years the parallel development of this method and ever improving mass-spectroscopy instrumentation will greatly facilitate efforts to improve our spatial understanding of the regulation of cellular metabolism.

Acknowledgements We would like to thank Britta Hausmann and Helga Kulka for careful supervision of greenhouse plants, Ute Roessner, Cornelia Wagner, and Katrin Bieberich for advice on GCMS measurements.

\section{References}

Bologa, K. L., Fernie, A. R., Leisse, A., Ehlers-Loureiro, M., \& Geigenberger, P. (2003). A bypass of sucrose synthase leads to low internal oxygen and impaired metabolic performance in growing potato tubers. Plant Physiology, 132, 2058-2072.

Benkeblia, N., Shinano, T., \& Osaki, M. (2007). Metabolite profiling and assessment of metabolome compartmentation of soybean leaves using non-aqueous fractionation and GC-MS analysis. Metabolomics, 3, 297-305. 
Chia, T., Thorneycroft, D., Chapple, A., Messerli, G., Chen, J., Zeeman, S. C., Smith, S. M., \& Smith, A. M. (2004). A cytosolic glucosyltransferase is required for conversion of starch to sucrose in Arabidopsis leaves at night. Plant Journal, 37, 853-863.

Etxeberria, E., Gonzalez, P., Tomlinson, P., \& Pozueta-Romero, J. (2005). Existence of two parallel mechanisms for glucose uptake in heterotrophic plant cells. Journal of Experimental Botany, 56, 1905-1912.

Farré, E. M., Tiessen, A., Roessner, U., Geigenberger, P., Trethewey, R. N., \& Willmitzer, L. (2001) Analysis of the compartmentation of glycolytic intermediates, nucleotides, sugars, organic acids, amino acids, and sugar alcohols in potato using a nonaqueous fractionation method. Plant Physiology, 127, 685-700.

Fernie, A. R., Riesmeier, J. W., Martiny, A., Ramalingan, S., Willmitzer, L., \& Trethewey, R. N. (2000). Consequences of the expression of a bacterial glucokinase in potato tubers, both in combination with and indepently of a yeast-derived invertase. Australian Journal of Plant Physiology, 27, 827-833.

Fernie, A. R., \& Willmitzer, L. (2001). Molecular and biochemical triggers of potato tuber development. Plant Physiology, 127, $1459-1465$.

Fernie, A. R., Roessner, U., \& Geigenberger, P. (2001) The sucrose analog palatinose leads to a stimulation of sucrose degradation and starch synthesis when supplied to discs of growing potato tubers. Plant Physiology, 125, 1967-1977.

Fernie, A. R., Trethewey, R. N., Krotzky, A. J., \& Willmitzer, L. (2004). Innovation - Metabolite profiling: from diagnostics to systems biology. Nature Reviews in Molecular Cell Biology, 5, 763-769.

Fettke, J., Eckermann, N., Poeste, S., Pauly, M., \& Steup, M. (2004). The glycan substrate of the cytosolic (Pho2) phosphorylase isozyme from Pisum sativum L.: identification, linkage analysis and subcellular localization. Plant Journal, 39, 933-946.

Fettke, J., Poeste, S., Eckermann, N., Tiessen, A., Pauly, M., Geigenberger, P., \& Steup, M. (2005). Analysis of cytosolic heteroglycans from leaves of transgenic potato (Solanum tuberosum L.) plants that under- or overexpress the Pho2 phosphorylase iosozyme. Plant Cell Physiology, 46, 1987-2004.

Gerrits, N., Turk, S. C. H. J., van Dun, K. P. M., Hulleman, S. H. D., Visser, R. G. F, Weisbeek, P. J., \& Smeekens, S. C. M. (2001). Sucrose metabolism in plastids. Plant Physiology, 125, 926-934.

Hajirezaei, M., Takahata, Y., Trethewey, R. N., Willmitzer, L., \& Sonnewald, U. (2000). Impact of elevated cytosolic and apoplastic invertase activity on carbon metabolism during potato tuber development. Journal of Experimental Botany, 51, 439445 .

Heineke, D., Willdenberger, K., Sonnewald, U., Willmitzer, L., \& Heldt, H. W. (1994) Accumulation of hexoses in leaf vacuoles studies with transgenic tobacco plants expressing yeast-derived invertase in the cytosol and vacuole or apoplasm. Planta, 194, 29-33.

Junker, B. H., Wuttke, R., Nunes-Nesi, A., Steinhauser, D., Büsses, D., Willmitzer, L., \& Fernie, A. R. (2006). Enhancing vacuolar sucrose cleavage within the potato tuber has only minor effects on metabolism. Plant Cell Physiology, 47, 277-289.

Kammerer, B., Fisher, K., Hilpert, B., Schubert, S., Gutensohn, M., Weber, A., \& Flugge, U. I. (1998). Molecular characterisation of a carbon transporter in plastids from heterotrophic tissues: The glucose 6-phosphate antiporter. Plant Cell, 10, 105-117.
Kaplan, F., Guy, C. L. (2005). RNA interference of Arabidopsis betaamylase8 prevents maltose accumulation upon cold shock and increases sensitivity of PSII photochemical efficiency to freezing stress. Plant Journal, 44, 730-743.

Kopka, J. (2006). Current challenges and developments in GC-MS based metabolite profiling technology. Journal of Biotechnology, 124, 312-322.

Leidreiter, K., Kruse, A., Heineke, D., Robinson, D. G., \& Heldt, H. W. (1995). Subcellular volumes and metabolite concentrations in potato (Solanum tuberosum cv. Desiree) leaves. Botanica Acta, 108, 439-444.

Lytovchenko, A., Sonnewald, U., \& Fernie, A. R. (2007). The complex network of non-cellulosic carbohydrate metabolism. Curr. Opin. Plant Biology, 10, 227-235.

Merlo, L., Geigenberger, P., Hajirezaei, M., \& Stitt, M. (1993) Changes in carbohydrates, metabolites and enzyme activities in potato tubers during development, and within a single tuber along a stolon-apex gradient. Journal of Plant Physiology, 142, 392-402.

Renz, A., Merlo, L., \& Stitt, M. (1993) Partial purification of three fructokinases and three hexokinases which show differing organ and developmental specificity. Planta, 190, 156-165.

Riens, B., Lohaus, G., Heineke, D., \& Heldt, H. W. (1991) Aminoacid and sucrose content determined in the cytosolic, chloroplastic, and vacuolar compartments and in the phloem sap of spinach leaves. Plant Physiology, 91, 227-233.

Roessner, U., Luedemann, A., Brust, D., Fiehn, O., Thomas, L., Willmitzer, L., \& Fernie, A. R. (2001) Metabolic profiling allows comprehensive phenotyping of genetically or environmentally modified plant systems. Plant Cell, 13, 11-29.

Roessner-Tunali, U., Liu, J., Leisse, A., Balbo, I., Perez-Melis, A., Willmitzer, L., \& Fernie, A. R. (2004). Kinetics of labelling of organic and amino acids in potato tubers by gas-chromatography-mass-spectrometry following incubation in 13C labelled isotopes. Plant Journal, 39, 668-679.

Sonnewald, U., Hajirezaei, M. R., Kossmann, J., Heyer, A., Trethewey, R. N., \& Willmitzer, L. (1997) Expression of a yeast invertase in the apoplast of potato tubers increases tuber size. Natural Biotechnology, 15, 794-797.

Sweetlove, L. J., Burrell, M. M., \& ap Rees, T. (1996). Characterization of transgenic potato (Solanum tuberosum) tubers with increased ADPglucose pyrophosphorylase. Biochemistry Journal, 320, 487-492.

Tiessen, A., Hendriks, J. H. M., Stitt, M., Branscheid, A., Gibon, Y., Farre, E. M., \& Geigenberger, P. (2002). Starch synthesis in potato tubers is regulated by post-translational redox modification of ADP-glucose pyrophosphorylase: a novel regulatory mechanism linking starch synthesis to the sucrose supply. Plant Cell, 14, 2191-2213.

Trethewey, R. N., Geigenberger, P., Riedel, K., Hajirezaei, M. R., Sonnewald, U., Stitt, M., Riesmeier, J. W., \& Willmitzer, L. (1998) Combined expression of glucokinase and invertase in potato tubers leads to a dramatic reduction in starch accumulation and a stimulation of glycolysis. Plant Journal, 15, 109-118.

Urbanczyk-Wochniak, E., Luedemann, A., Kopka, J., Selbig, J., Roessner-Tunali, U., Willmitzer, L., \& Fernie, A. R. (2003). Parallel analysis of transcript and metabolite profiles: a new approach in systems biology. EMBO reports, 4, 989-993.

Weise, S. E., Weber, A. P., \& Sharkey, T. D. (2004). Maltose is the major form of carbon exported from the chloroplast at night. Planta, 218(3), 474-482. 